\title{
Experiences of Hospice and Palliative Nurses in Response to the COVID-19 Pandemic: A Qualitative Study
}

\author{
Sinyoung Kwon, A.P.N., Ph.D. and Sujin Choi, R.N., Ph.D.* \\ Department of Nursing, Gangdong University, Eumseong, ${ }^{*}$ College of Nursing, Woosuk University, Wanju, Korea
}

Purpose: This study aimed to explore the experiences of hospice and palliative care (HPC) nurses at inpatient hospice centers in South Korea during the coronavirus disease 2019 pandemic. Methods: Data collection was conducted through individual interviews with 15 HPC nurses using face-to-face interviews, telephone calls, or Zoom videoconferencing. Data were analyzed using the thematic analysis method. Results: This study found that HPC nurses experienced practical and ethical dilemmas that reinforced the essential meaning and value of hospice and palliative care. The participants emphasized their practical roles related to compliance with infection prevention measures and their roles as rebuilders of hospice and palliative care. Conclusion: The findings of this study indicate that inpatient hospice centers must mitigate the practical and ethical dilemmas experienced by nurses, consider establishing explanation nursing units, and provide education to support nurses' highlighted roles during the pandemic. This study can be used to prepare inpatient hospice centers and the nurses that work there for future infectious disease outbreaks.

Key Words: COVID-19, Palliative care, Hospice care, Hospice and palliative care nursing
Received August 23, 2021

Revised October 16, 2021

Accepted October 30, 2021

Correspondence to

Sujin Choi

ORCID:

https://orcid.org/0000-0002-7440-6551

E-mail:schoi@woosuk.ac.kr

\section{INTRODUCTION}

Hospice and palliative care (HPC) is based on a multidisciplinary approach for providing care that is tailored to the values of patients and families by helping end-stage patients with overall pain relief, improving their quality of life, and optimizing comfort [1]. In particular, inpatient HPC services are often aimed at terminal cancer patients, in addition to their families, who are admitted to the hospice unit of a specialized medical institution designated by the Ministry of Health and Welfare [2]. Such specialized institutions provide patients with hospice and palliative care services to maintain a dignified life.

Following the worldwide outbreak of coronavirus disease 2019 (COVID-19), as of April 23, 2021, the COVID-19 infection rate in South Korea has increased, with 117,458 con- firmed cases [3]. Although the second wave of the pandemic has passed, outbreaks in hospitals and nursing homes have been reported [4]. Accordingly, social distancing measures have been reinforced and hospitals with hospice centers have implemented regulations to prevent the spread of infectious diseases within them, thereby restricting access to visitors, such as family caregivers [5,6]. For example, hospice centers limited the number of visiting family members that could be at the bedside of patients in the event of death, according to the guidelines of the Ministry of Health and Welfare [5].

HPC is available to people of all ages [7]. Therefore, its demand is projected to increase due to the number of severely ill patients in the wake of the COVID-19 pandemic. Additionally, as HPC is predicted to play a key role in medical institutions in the response to the COVID-19 pandemic $[8,9]$, 
academic studies of clinical experience have been published internationally, including studies that have presented advanced guidelines for providing high-quality services while maintaining the dignity of patients at the end of life. However, the subject is still underexamined in South Korea. Additionally, inpatient hospice centers mainly treat patients with terminal cancer who are more susceptible to COVID-19 due to their low immunity and high risk of infection; therefore, it is necessary to investigate how HPC is administered in the context of the COVID-19 pandemic.

Among healthcare professionals who provide HPC, nurses are on the frontlines of delivering safe and comfortable palliative care to patients in need [10]. As nurses have typically been most aware of changes in hospital operations as well as of the responses of patients and their families to the COVID-19 pandemic, the experiences and perceptions of nurses at inpatient HPC centers must be explored. Given the clinical impact of the COVID-19 pandemic on HPC, its increasing demand, and the characteristics of patients, academic inquiries from the perspectives of nurses perspectives are needed. Such studies will, in turn, provide evidence for leading to further refinements of practical guidelines for administering HPC during the pandemic. Therefore, this study was conducted to explore the experiences of nurses at inpatient hospice centers during the COVID-19 pandemic. The specific research questions were (1) "How do nurses who work at inpatient hospice centers view the COVID-19 pandemic?" and (2) "What changes in HPC have nurses observed during the COVID-19 pandemic?"

\section{METHODS}

This study used a qualitative research design with the thematic analysis method to examine the experiences of nurses who work in inpatient hospice centers during the COVID-19 pandemic. Due to its theoretical freedom and flexibility as a research tool, the thematic analysis method is widely used in qualitative research [11]. The authors unanimously approved the use of the thematic analysis method.

\section{Participants}

The inclusion criteria for the participants in this study were active nurses who had worked for more than 3 years as a nurse at an inpatient hospice center, since the authors perceived that nurses with more than 3 years of experience had a superior understanding of the overall situation and context than nurses with less experience, making it possible for the authors to tell a rich story from various experiences. The participants understood the purpose and voluntarily participated in this study.

\section{Data collection}

Data were collected from December 24, 2020, to January 5, 2021, after receiving approval from the Institutional Review Board (IRB No.: KIRAMS 2020-12-003). Using the convenience sampling method, the first author contacted administrators at six inpatient hospice centers with palliative care units in Seoul, Gyeonggi Province, and both South and North Jeolla Provinces and explained the purpose of the study. The administrators then voluntarily introduced the first author to participants who agreed to share their contact information. The first author called the participants and asked them to sign an informed consent form online after explaining the purpose of the study and the protocols related to the protection of personal information. Once the authors received and verified the signed informed consent form, interviews were scheduled according to the preferences of the participants. A total of 15 participants (9 from Seoul, 2 from Gyeonggi Province, and 4 from Jeolla Province) were included. Face-to-face interviews were conducted with the consent of the participants who worked at the same institution as the first author. For the participants who worked at other institutions, interviews were conducted using Zoom videoconferencing. Each interview lasted 30 to 65 minutes. Data saturation was achieved after 15 interviews with 15 participants.

\section{Data analysis}

Thematic analysis was conducted in six stages according to the method outlined by Braun and Clarke [11]. The first stage was for the researchers to familiarize themselves with the data. In this study, the authors fully transcribed the interviews immediately after each interview. By reading the transcripts repeatedly, the authors gained familiarity with the data. The second stage was to identify meaningful codes that appeared repeatedly. The third stage was to create subcategories that 
reflected the meaning of the raw data. The fourth stage was to re-examine and revise the previously derived subcategories. The fifth stage was to create further-refined categories that were clear and consolidated. The sixth and final stage of the study involved reviewing the most meaningful codes.

\section{Ethical considerations}

The participants were allowed to withdraw or refuse to participate at any time during or at the beginning of the interview or to change the topic during the interview. The participants were also assured that if they withdrew their consent, their data would not be used for research. In addition, they were informed that the collected data would not be used for any other purpose except for this study. Moreover, the participants were assured of their anonymity and confidentiality throughout the study and that the data would be accessible only to the authors of the study.

\section{Rigor of research}

The authors attempted to ensure the quality of this study using the criteria by Lincoln and Guba [12] for evaluating the rigor of a study: credibility, dependability, confirmability, and transferability. To ensure credibility, we verified that the findings of this study were true, and the data underwent a peer debriefing with two outside qualitative researchers during the analysis stage. To ensure dependability, field notes were recorded during data collection. To ensure confirmability, the data collection and analysis methods were transparently illustrated, and the researchers re-listened to the recorded audio files of interviews throughout the entire study process. To ensure transferability, meaningful experiences and their full contexts were described in detail in order to fully reflect the experiences of the HPC nurses.

\section{RESULTS}

\section{General characteristics of participants}

The participants in this study were 15 female nurses who worked at inpatient hospice centers. The average age of the participants was 38 years old (range: 26 52 years old). Seven nurses had a Master of Science in Nursing degree, 1 nurse had a graduate degree, 6 nurses had a Bachelor of Science in Nursing degree, and 1 nurse had a Nursing diploma. Their average nursing experience was 14 years and 3 months, and their average HPC nursing experience was 8 years and 7 months.

\section{Experiences of hospice and palliative care nurses during the COVID-19 pandemic}

Using qualitative thematic analysis [11], units of meaning were extracted related to HPC nurses' experiences responding to the COVID-19 pandemic. Based on the units of meaning, 7 subcategories and 3 categories were identified (Table 1).

\section{1) The first category: dilemmas}

The first category, "dilemmas," refers to the difficulties experienced by nurses who provided care for patients during the COVID-19 pandemic. Its subcategories were "practical dilemmas related to wearing masks" and "ethical dilemmas related to caring."

\section{(1) Practical dilemmas related to wearing masks}

The participants had difficulty with communication while caring for inpatient hospice patients. According to the COVID-19 guidelines, all medical personnel and patients had to wear masks. However, masks made it difficult for nurses to observe changes in patients' facial expressions or identify sub-

Table 1. Categories and Subcategories of Experiences of Hospice and Palliative Care Nurses during the COVID-19 Pandemic.

\begin{tabular}{ll}
\hline \multicolumn{1}{c}{ Categories } & \multicolumn{1}{c}{ Subcategories } \\
\hline Dilemmas & Practical dilemmas related to wearing masks \\
& Ethical dilemmas related to caring \\
Strengthening the basic meaning and value of hospice and palliative care & Reinforcement of one's awareness of human dignity \\
& Back to the basics \\
Highlighting the role of nurses & Enhancing the role of explanation \\
& Consolidating the role of supporting patients and families \\
& Reinforcing the role of devising strategies \\
\hline
\end{tabular}


tle emotions when talking with patients, as well as to provide emotional support to patients. Considering the general characteristics of patients at inpatient hospice centers, many patients had poor hearing, and the participants felt sorry for patients who experienced difficulties understanding what the nurses were saying to them since they could not look at the nurses' mouths. Moreover, the nurses found it difficult to understand conversations when discussing patient treatment plans with other medical personnel who were wearing masks.

All the patients except those with dyspnea had to wear masks. However, I felt sorry that I could not check for changes in the patients' facial expressions or subtle emotions. It is even more difficult because there are many patients whose mental status changes from drowsy to a stupor. Managing patients' emotions is an essential element of holistic nursing. (Participant 11)

When caring for elderly patients, they and their family caregivers often have amblyacousia. Before COVID-19, they could understand from the shape of my mouth and by speaking loudly. However, now I wear a mask, and they cannot look at my mouth and facial expressions. It is very difficult to communicate. (Participant 4)

When physicians make rounds, I stand behind them. I could not look at the physician's facial expression, and I could not understand their therapeutic conversation well. Patients also could hardly understand the physician as we all wore masks. (Participant 9)

\section{(2) Ethical dilemmas related to caring}

The main duty of nurses is to take care of patients, but this was the first time the nurses faced something like the COVID-19 pandemic. The participants continued going to work with the patients in mind despite fears related to the spread of COVID-19. They also often felt overwhelmed while dealing with family caregivers who complained about shortened visiting hours and strict visiting limitations. However, rather than focusing on fear and feeling overwhelmed, they felt sad that patients and families could not see each other and experience a dignified death. Regarding family caregivers who lived abroad, the nurses felt pity for those who had to travel to South $\mathrm{Ko}^{-}$ rea and undertake complicated procedures due to pandemicrelated regulations such as spending 2 weeks in isolation before the patient's death. The participants also experienced ethical distress as they sometimes felt cold-hearted due to their enforcement of regulations.

Honestly, when I go to work, I feel scared: partly afraid, partly concerned for the patients... Today, I am doing the work with a heavy emotional load and focusing on how to take care of patients while practicing social distancing and quarantine. (Participant 6)

Besides getting overburdened, I think the death of patients is the most painful thing to experience as a nurse during the COVID-19 pandemic... This is a dilemma for nurses. Family caregivers came to see the patient and came up through the control office on the first floor, but I had to say, "No visiting." Am I doing the right thing for the patient? I feel like I am a bad nurse. Such a visit could be the last visit for the patients and family caregivers. (Participant 2)

There were cases in which patients could not wait 2 weeks for family caregivers coming from abroad. The patient's condition was getting worse. The nurse and the family caregivers were agitated. After the isolation period, the family caregiver could visit the patient but by then the patient could have passed away. (Participant 4)

\section{2) The second category: strengthening the basic meaning and value of hospice and palliative care}

The second category refers to reinforcement of the basic meaning and value of HPC that nurses experienced during the COVID-19 pandemic. The subcategories were "reinforcement of one's awareness of human dignity" and "back to the basics."

\section{(1) Reinforcement of one's awareness of human dignity}

The participants stated that the core meaning and value of HPC is to provide nursing care with love so that the patient feels special and nurtured. It was commonly identified that focusing on patient comfort, which is the core meaning and value of HPC, had not changed due to the pandemic. Rather, 
patient comfort was even more crucial during the pandemic. It was important for the nurses to consider the quality of life at the moment of death instead of just focusing on the outbreak of an infectious disease. The participants highlighted that the goal of HPC had not changed; therefore, efforts to reorganize the surrounding environments of patients were also necessary to prepare for the post-COVID-19 era.

I think the patient dying well is the optimal goal. Furthermore, COVID-19 does not seem to change the meaning and value of HPC. I wish the nursing care and services we provided were similar to the former nursing care and services. (Participant 9)

The changed nursing practices corresponding to the pandemic do not focus on infectious diseases. In this situation, the best thing is to focus on the quality of life of patients in the process of dying. (Participant 3)

\section{(2) Back to the basics}

Although the participants were not usually aware of basic measures previously, many said that they identified an emphasis on basic nursing practices during the COVID-19 pandemic. Among such basic practices, visitor management, wearing masks, thorough hand washing, and environmental management such as cleaning and quarantine procedures were supposed to be observed even before the COVID-19 pandemic. However, the pandemic posed an opportunity for nurses to reaffirm the importance of such basic practices again. Thus, the participants mentioned that both patients and medical personnel should follow basic measures. Additionally, despite the apparent triviality of basic tasks, most of which tended not to have a significant effect on the participants' nursing performance, such tasks still helped patients to experience a dignified death in a comfortable environment.

There is no medicine for COVID-19; the most important and basic measures to practice are wearing a mask and washing hands. Everyone should practice these measures daily at work. I did not think it was important, but the most important thing now is practicing these basic measures (Participant 2).
Perhaps it's like wiping the patients' eyes, or even the smallest things, such as cleaning their eyelids and grabbing their hands. Nowadays, when I provide HPC to a patient at the end of life, I pray before administering nursing care, "You [the patient] did a lot of hard work, and you deserve to go to a good place to rest" (Participant 10).

\section{3) The third category: highlighting the role of nurses}

The third category refers to the reaffirmation of the existing roles of nurses during the COVID-19 pandemic and the subsequent rediscovery of various roles required of nurses. The subcategories were "enhancing the role of explanation," "consolidating the role of supporting patients and families," and "reinforcing the role of the devising strategies."

\section{(1) Enhancing the role of explanation}

Restricting visitors made communication between nurses, $\mathrm{pa}^{-}$ tients, and families difficult. Usually, nurses helped the families of patients understand the patients' conditions by explaining to them who visited them and answering questions that arose after end-of-life counseling. As the COVID-19 pandemic progressed, the participants were confronted with an increased need to explain the hospitalization process and guidelines to families and experienced difficulties with face-to-face consultations. The participants sometimes even had to explain patients' conditions to family members over the phone due to concerns about distorted information. Additionally, only a limited number of family members could be present upon patients' deaths. The moment of a patient's death was shared over the phone with family members who could not attend so that they could listen to the nurse's explanation together. As such, the role of explanation to improve the quality of terminal cancer patients' deaths was emphasized for the participants. They became aware of the need to hone their communication skills to effectively relay important information.

The work increased insignificantly. The need to provide explanations, however, seems to have increased. I instructed the patients and family caregivers to do a temperature check twice a day and fill out a guestbook despite completing the COVID-19 checklists and temperature check at the entrance. (Participant 7) 
We limit visitors and call the patient's family occasionally when the patient's condition changes. If the primary family caregiver was the eldest daughter, I called her and then other siblings, respectively. This is because delivering information over the phone is different when non-medical personnel and medical personnel deliver information. (Participant 1)

\section{(2) Consolidating the role of supporting patients and families}

Only one caregiver was appointed per patient in inpatient hospice centers during the pandemic. The caregiver could be a family caregiver who had to live in quarantine with the patient. The participants mentioned that it was necessary to support the appointed family caregivers who were burdened with care and ensure that they did not suffer emotionally and could take good care of the patient. Additionally, as family members other than the appointed family caregiver were restricted from visiting, the participants came to understand the importance of the role of supporters so that patients did not experience loneliness leading up to the moment of their deaths.

The primary family caregiver may feel more aggravated because only one caregiver could be present, even during the moment of a patient's death. If they were with another caregiver, they could communicate and rely on each other, but that is impossible currently. It is very burdensome to care for patients alone in a single room. They need emotional support (Participant 13).

Some family members thought that the patients were having a hard time not seeing the people they wanted to see. I think family is more like that. COVID-19 cannot be eliminated now. Therefore, I think it is important that nurses help patients not feel lonely (Participant 15).

\section{(3) Reinforcing the role of devising strategies}

The participants agreed that it was necessary to consider patient education and provide support meetings to address family bereavement using non-face-to-face means, rather than just reacting and seeking to provide comfort to patients in the COVID-19 pandemic. In fact, due to visiting limitations, the participants suggested specific methods, such as helping the patients make video calls with family members who could not meet each other. Additionally, due to the limited number of visitors and shortened visiting time, family members could not visit patients frequently. As a result, the participants had to consider how to effectively coordinate visiting time for patients and their families. The participants recognized the necessity of redesigning hospice services to accommodate non-face-toface means of communication.

In the long run, it is time to consider using digital equipment. I also thought that caring for patients was the role of a nurse. Now, nurses need to broaden their scope and provide care via non-face-to-face means. (Participant 4)

Due to visiting limitations, I recommended video calls to patients who wanted to see and meet their grandchildren. Listening to the voice of their grandchildren contributed to positive changes in conscious or unconscious patients such as trembling in their eyebrows and laughter. (Participant 2)

I feel like I need to develop something that can be used for hospice care in non-face-to-face situations. Later [postCOVID-19 pandemic], volunteers [different multidisciplinary teams] will return to the hospital. Then, hospice care plans for non-face-to-face situations should be rebuilt. (Participant 14)

\section{DISCUSSION}

This study described the experiences of HPC nurses at inpatient hospice centers during the COVID-19 pandemic. This study identified 3 categories of pandemic-related experience for HPC nurses. The first category referred to practical dilemmas caused by the obligatory use of masks. Although the challenges of communication following the mask mandate in hospitals are under-reported in the current literature, initial communication between high-risk patients and healthcare providers regarding the goals of care and the end of life has never been more critical than during the pandemic [7]. Communication has often been limited to patients during the pandemic due to limitations to face-to-face clinical interaction [6]. Healthcare providers have recognized the need for a focused plan and developed crisis standards of care for HPC [13]. When developing standards of care, the findings of this study 
should be used to consider the practical challenges of communication resulting from mask mandates at HPC facilities. Future studies should examine the implementation of different categories of risk for patients related to masks, such as patients for whom masks are mandated, recommended, or not recommended depending on their condition and the environment of the HPC unit, which could enhance the quality of communication as well as the quality of HPC overall.

Ethical dilemmas were identified as another issue. As was also reported by nurses in other countries, the nurses in the present study feared the rapid spread of COVID-19 [14]; however, they continued to go to work to care for patients while simultaneously feeling cold-hearted due to their roles strictly enforcing visiting precautions and restrictions, thus inhibiting interaction between family members and patients at the end of life. This finding is similar to that of a study that investigated the effect of social distancing measures on the emotional wellbeing of patients and their families, as well as staff members [15]. The study found that the most common barrier to HPC during the pandemic was strict visitation policies for family caregivers at hospitals, describing the heartbreak of seeing family members crying in full personal protective equipment. As such, COVID -19 has raised ethical dilemmas across all care levels, and ethical dilemmas have become apparent in the context of many morally distressing clinical scenarios [16]. It is important to note that in South Korea, family involvement and support are customary when patients are admitted to the hospital. Family caregivers were permitted and even encouraged to stay at patients' bedsides and participate in patients' care before the pandemic. Patients relied heavily on primary family caregivers for trivial needs as well [17]. Given the cultural context, HPC nurses in South Korea are likely to experience more significant ethical dilemmas related to visiting limitations for family visitors and patients at the end of life. Therefore, instead of burdening HPC nurses with the need to confront ethical conflicts, hospice centers can assess the physical and psychological symptoms caused by ethical dilemmas to further provide individual interventions related to such symptoms.

The third category was the emphasis on the nurse's role in providing explanations, supporting patients and family, and devising strategies. Kates et al. [15] similarly examined the impact of COVID-19 on the HPC workforce and identified an absence of "real-time explanation and education," emphasizing the need for facilitating more time for patients and families to communicate. The COVID-19 guidelines also highlighted education related to COVID-19 symptoms and the treatment of patients, families, and healthcare workers [9]. Given nurses' enhanced role in providing explanations during the COVID-19 pandemic, it must be ensured that HPC nurses provide thorough and situation-appropriate explanations to patients and families. A study by Yoo [18] described the positive effect of an explanation nursing unit on patient satisfaction by providing explanations about the purpose of procedures, the process, the interpretation of procedures' results, and precautions before and after procedures. Hospice centers should consider establishing explanation nursing units for the purpose of providing additional explanations about procedures and care plans as well as the hospitalization experience during the pandemic.

Concerning the role of nurses in making strategies, this study described the increased role of nurses in rebuilding HPC services in response to this new era. The most significant issue affecting families during the pandemic was impaired communication. This was mainly reported as miscommunication between family caregivers and healthcare personnel and the inability of patients to communicate with their family networks [19]. Healthcare providers must change their practices in response to the global pandemic. Some examples included holding all family meetings over the phone, conducting all patient visits via video conference or phone, and facilitating communication via a listserv to update families [9]. Such changes in providing care offer opportunities for nurses to be creative with patient interactions and services [19]. There is an urgent need to develop education to support the role of nurses as rebuilders of HPC in the wake of the new normal. It is recommended that continuing education and in-service training for nurses include educational programs on new methods, tools, and strategies for communication and education.

Despite the emphasis on the important role of HPC in times of humanitarian crises and pandemics [9], the global response to COVID-19 exposed a lack of efficient protocols for HPC during a pandemic and gaps in training and literature related to palliative care $[8,20]$. In this study, HPC nurses provided service to patients while confronting practical and ethical di- 
lemmas, recognizing the importance of HPC and the need to enhance their roles in explaining, supporting, and rebuilding nursing services in response to the new normal. The findings of this study encourage inpatient hospice centers to take actions to mitigate practical dilemmas, assess ethical dilemmas experienced by HPC nurses, implement explanation nursing units, and provide education to HPC nurses so that they can overcome dilemmas and fully inhabit their reinforced roles during the pandemic. The results of this study can be used as practical data for hospice centers and the nurses working there to prepare for future infectious disease outbreaks.

\section{CONFLICT OF INTEREST}

No potential conflict of interest relevant to this article was reported.

\section{ORCID}

Sinyoung Kwon, https://orcid.org/0000-0001-8707-9281

Sujin Choi, https://orcid.org/0000-0002-7440-6551

\section{AUTHOR'S CONTRIBUTIONS}

Conception or design of the work: all authors. Data collection: SK. Data analysis and interpretation: all authors. Drafting the article: all authors. Critical revision of the article: all authors. Final approval of the version to be published: all authors.

\section{SUPPLEMENTARY MATERIALS}

Supplementary materials can be found via https://doi. org/10.14475/jhpc.2021.24.4.245.

\section{REFERENCES}

1. Rosa WE, Finlayson CS, Ferrell BR. The cancer nurse as primary palliative care agent during COVID-19. Cancer Nurs 2020;43:431-2.

2. Korea Ministry of Health and Welfare. 2021 Notice for hospice palliative care services [Internet]. Sejong: Ministry of Health and Welfare; 2021 [cited 2021 Aug 19]. Available from: http://www.mohw.go.kr/react/jb/sjb030301 vw.jsp?PAR_MENU_ID=03\&MENU_ ID $=032901 \&$ CONT SEQ $=363986$.

3. Korea Centers for Disease Control and Prevention. The current status of COVID-19 confirmed cases [Internet]. Cheongju: Korea Disease \& Prevention Agency; 2021 [cited 2021 Apr 23]. Available from: http://ncov.mohw.go.kr/bdBoardList_Real.do? brdld=1\&brdGubun=11\&ncv ContSeq=contSeq=\&board_id=\&gubun= Accessed ib.

4. Lim S. The COVID-19 pandemic battlefield: protection of long term care hospitals. Infect Chemother 2020;52:231-3.

5. Korea Ministry of Health and Welfare. Outbreak of infectious disease: guidelines for operating hospice specialized institutions [Internet]. Sejong: Ministry of Health and Welfare; 2020 [cited 2020 Oct 20]. Available from: https://hospice.go.kr/pain/archivesView.do? brd_no=98913.

6. Abbott J, Johnson D, Wynia M. Ensuring adequate palliative and hospice care during COVID-19 surges. JAMA 2020;324:1393-4.

7. Koch A, Mantzouris S. Nurses' role in providing comprehensive communication, prognostication, and palliative care during the COVID-19 pandemic. J Hosp Palliat Nurs 2020;22:442-6.

8. Etkind SN, Bone AE, Lovell N, Cripps RL, Harding R, Higginson IJ, et al. The role and response of palliative care and hospice services in epidemics and pandemics: a rapid review to inform practice during the COVID-19 pandemic. J Pain Symptom Manage 2020;60:e31-e40.

9. Gilissen J, Pivodic L, Unroe KT, Van den Block L. International COVID-19 palliative care guidance for nursing homes leaves key themes unaddressed. J Pain Symptom Manage 2020;60:e56-e69.

10. Pai RR, Nayak MG, Sangeetha N. Palliative care challenges and strategies for the management amid COVID-19 pandemic in india: perspectives of palliative care nurses, cancer patients, and caregivers. Indian J Palliat Care 2020;26(Suppl 1):S121-S125.

11. Braun V, Clarke V. Using thematic analysis in psychology. Qual Res Psychol 2006;3:77-101.

12. Lincoln YS, Guba EG. Naturalistic inquiry. Beverly Hills, CA:Sage;1985.

13. CDPHE: Colorado contingency and crisis standards of care-palliative care and hospice services [Internet]. Glendale, CO: Colorado Department of Public Health and Environment; 2021 [cited 2021 Aug 18]. Available from: https://www.colorado.gov/pacific/cdphe/coloradocrisisstandards-care.

14. Wallace CL, Wladkowski SP, Gibson A, White P. Grief during the COVID-19 pandemic: considerations for palliative care providers. J Pain Symptom Manage 2020;60:e70-e76.

15. Kates J, Gerolamo A, Pogorzelska Maziarz M. The impact of COVID 19 on the hospice and palliative care workforce. Public Health Nurs 2021;38:459-63. 
16. Rosa WE, Meghani SH, Stone PW, Ferrell BR. Opportunities for nursing science to advance patient care in the time of COVID 19: a palliative care perspective. J Nurs Scholarsh 2020;52: 341-3.

17. Kang KA, Kim SJ. Comparison of perceptions of spiritual care among patients with life-threatening cancer, primary family caregivers, and hospice/palliative care nurses in South Korea. J Hosp Palliat Nurs 2020;22:532-51.

18. Yoo HH, Kim HM, Bae MH, Kim MJ, Woo Jl, Lee EY. Improvement of customer satisfaction with “Explanation Nurse Unit”. Qual Improv Health Care 2009;15:79-87.

19. Harden K, Price DM, Mason H, Bigelow A. COVID-19 shines a spotlight on the age-old problem of social isolation. J Hosp Palliat Nurs 2020;22:435-41.

20. Bolt SR, van der Steen JT, Mujezinovic I, Janssen DJ, Schols JM, Zwakhalen SM, et al. Practical nursing recommendations for palliative care for people with dementia living in long-term care facilities during the COVID-19 pandemic: a rapid scoping review. Int J Nurs Stud 2021;113:103781. 